\title{
A Fully Differential Monolithic 2.4GHz PA for IEEE 802.15.4 based on Efficiency Design Flow
}

Rafaella Fiorelli

Eduardo Peralías

IMSE-CNM CSIC-Univ. de Sevilla

Spain
Nicolás Barabino

Fernando Silveira
GME-Univ. de la República

Uruguay 


\section{Outline}

1. Introduction

2. PA modeling

3. PA Efficiency design flow

4. Circuit Implementation

5. Measurements

6. Conclusions and future work. 


\section{Introduction}

- An optimized class C PA suited for IEEE 802.15.4 is presented.

- Class C PAs support phase modulation.

- The design is being used to evaluate the Classes A-B-C PA design methodology [1].

[1] N. Barabino, R. Fiorelli, and F. Silveira, "Efficiency based design for fully-integrated class C RFpower amplifiers in nanometric CMOS" in IEEE International Symposium on Circuits and Systems (ISCAS), 2010. 


\section{PA modeling}

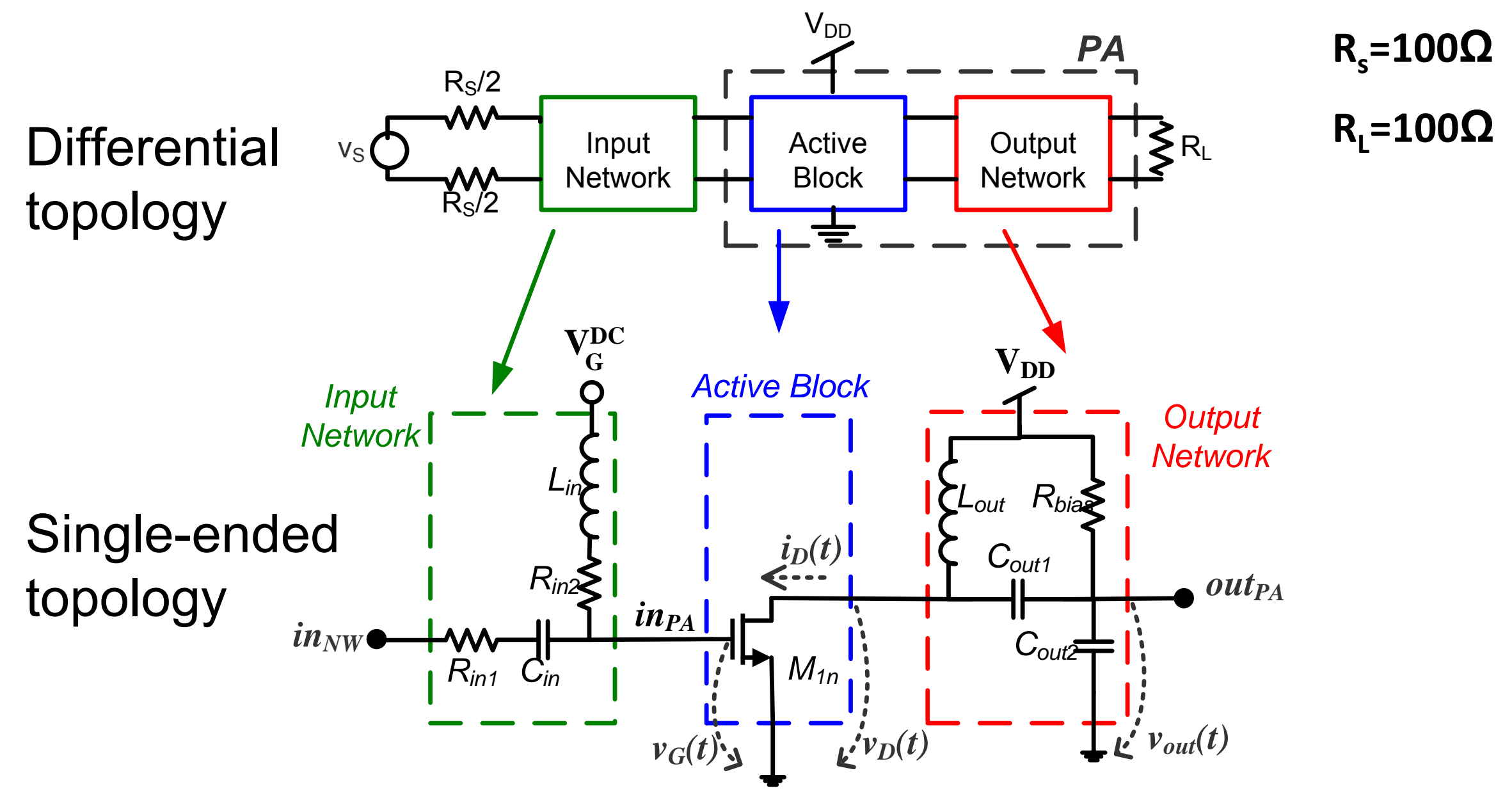




\section{PA design flow}

(1) MOS model in DC:

$\hat{\mathrm{i}}=\mathrm{I}_{D} /(\mathrm{W} / \mathrm{L})$ vs $\left(\mathrm{V}_{G}, \mathrm{~V}_{D}\right)$

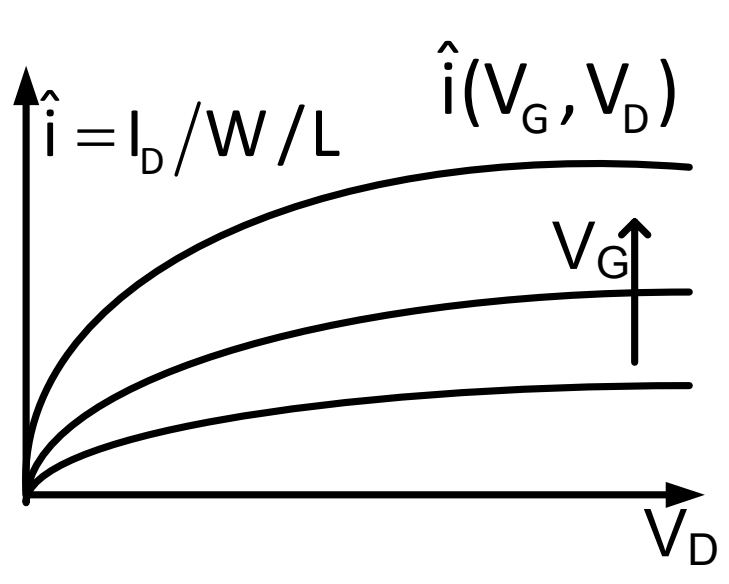

(2) Inductor modeling with S-parameters: $L_{\text {ind }}, Q, R_{p} @ f_{0}$

(3) Output NW: (i) transfer power to RL

(ii) filter the harmonics

$R_{L} \rightarrow R_{N W}$ (seen at the drain of MOS)

$$
\left\{\begin{array}{l}
P_{\text {out }}=\frac{V_{\text {out }}^{2}}{2 R_{L}} \cong \frac{V_{D}^{R F^{2}}}{2 R_{N W}} \\
V_{D}(t)=V_{D D}+V_{D}^{R F} \sin \left(\omega_{0} t\right)
\end{array}\right.
$$




\section{PA design flow}

(4) Choose $\left(V_{G}^{D C}, V_{G}^{R F}\right) \rightarrow v_{G}(t)=V_{G}^{D C}+V_{G}^{R F} \sin (\omega t)$

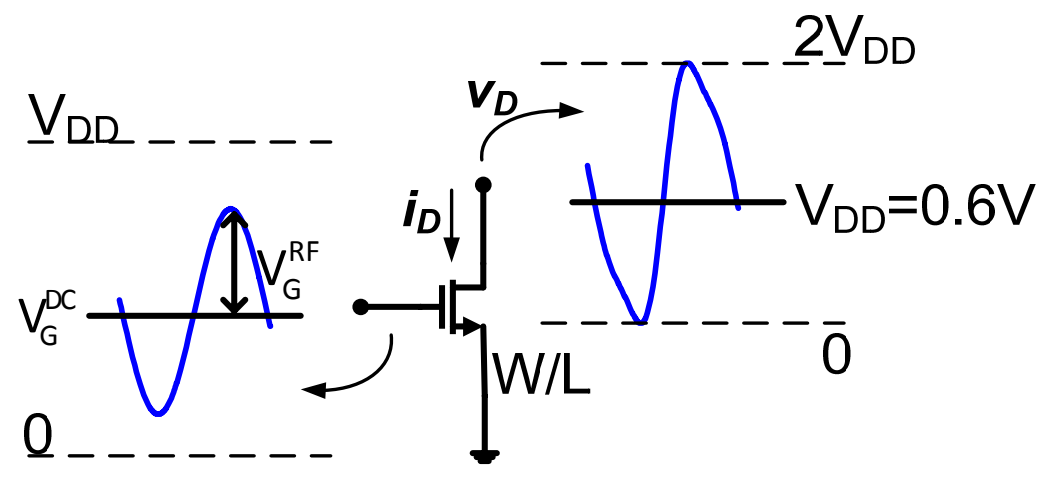

(5) Main hipothesis: $\hat{i}(t)$ obtained with $\hat{i}\left(V_{G}, V_{D}\right)$, the DC normalized current of step (1)

By Fourier, $\hat{l}_{D C}, \hat{l}_{1}, \hat{l}_{2}$ and $\hat{l}_{3}$ are calculated.
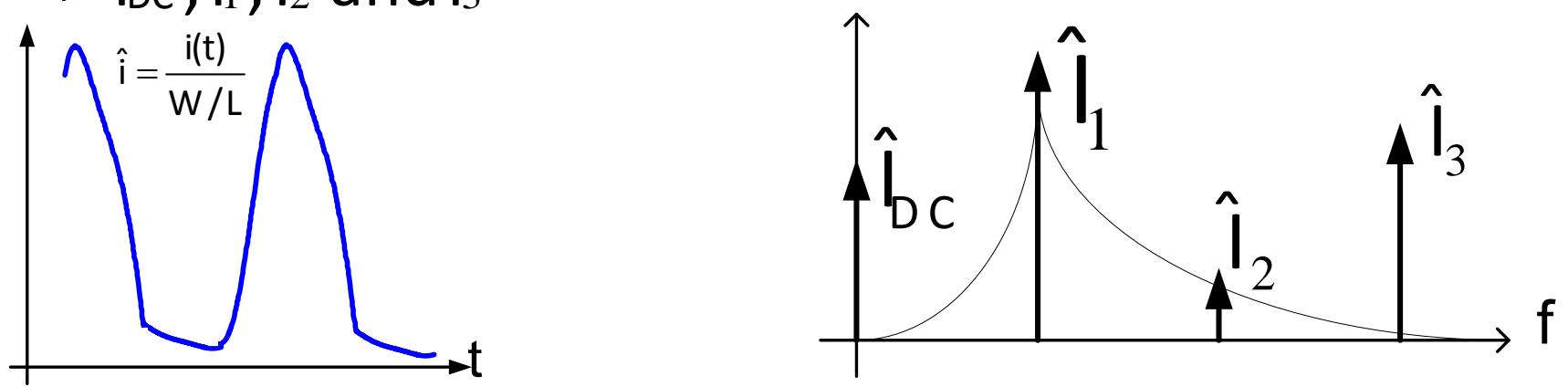


\section{PA design flow}

(6) Calculate the efficiency of MOS: $\eta_{\mathrm{MOS}}=\frac{\mathrm{P}_{\mathrm{NW}}}{\mathrm{P}_{\mathrm{DC}}}=\frac{\mathrm{I}_{1} \mathrm{~V}_{\mathrm{D}}^{\mathrm{RF}} / 2}{\mathrm{I}_{\mathrm{DC}} \mathrm{V}_{\mathrm{DD}}}=\frac{\hat{\mathrm{l}}_{1} \mathrm{~V}_{\mathrm{D}}^{\mathrm{RF}}}{2 \hat{\mathrm{l}}_{\mathrm{DC}} \mathrm{V}_{\mathrm{DD}}}$

(7) Calculate the misspent power of the output network and the total PA efficiency:

$\mathrm{P}_{\text {out }}=\mathrm{P}_{\mathrm{NW}}-\mathrm{P}_{\mathrm{miss}}=\frac{\mathrm{V}_{\mathrm{D}}^{\mathrm{RF}^{2}}}{2 \mathrm{R}_{\mathrm{NW}}}-\frac{\mathrm{V}_{\mathrm{D}}^{\mathrm{RF}^{2}}}{2 \mathrm{R}_{\mathrm{p}}} \rightarrow \eta_{\mathrm{NW}}=\frac{\mathrm{P}_{\text {out }}}{\mathrm{P}_{\mathrm{NW}}}=1-\frac{\mathrm{R}_{\mathrm{NW}}}{\mathrm{R}_{\mathrm{p}}} \rightarrow \eta=\eta_{\mathrm{MOS}} \eta_{\mathrm{NW}}$

For $P_{\text {out }}$ and $L_{\text {out }}$ solve for: $R_{N W}, P_{N w}$ and $\eta$. $L_{\text {out }}$ provides the correct harmonic filtering.

(8) Calculate the transistor aspect ratio: $\frac{W}{L}=\frac{l_{1}}{\hat{l}_{1}}=\frac{2 P_{N W} / V_{D}^{R F}}{\hat{l}_{1}}$ 


\section{Circuit Implementation}

\section{Summary of specifications}

\begin{tabular}{|l|l|l|}
\hline $\mathrm{P}_{\text {out }}>0 \mathrm{dBm}$ & $\mathrm{R}_{\mathrm{L}}=100 \Omega$ & $\mathrm{P}_{\text {harm }}^{2^{\text {nd }}} \leq-40 \mathrm{dBm}$ \\
\hline $\mathrm{V}_{\mathrm{D}}^{\mathrm{RF}}=0.6 \mathrm{~V}$ & $\mathrm{R}_{\mathrm{P}}>1 \mathrm{k} \Omega$ & $\mathrm{P}_{\text {harm }}^{3^{\text {nd }}} \leq-20 \mathrm{dBm}$ \\
\hline $\mathrm{V}_{\mathrm{DD}}=0.65 \mathrm{~V}$ & $\eta>35 \%$ & $\mathrm{P}_{1 \mathrm{~dB}} \geq-10 \mathrm{~dB}$ \\
\hline
\end{tabular}

1.2V RF 90nm CMOS technology, 1P9M.
Minimum $V_{G}$ supported by the technology limits us to select points with higher efficiency.

Final design point:

$$
\mathrm{V}_{\mathrm{G}}^{\mathrm{DC}}=0.5 \mathrm{~V}
$$$$
\mathrm{V}_{\mathrm{G}}^{\mathrm{RF}}=0.4 \mathrm{~V}
$$
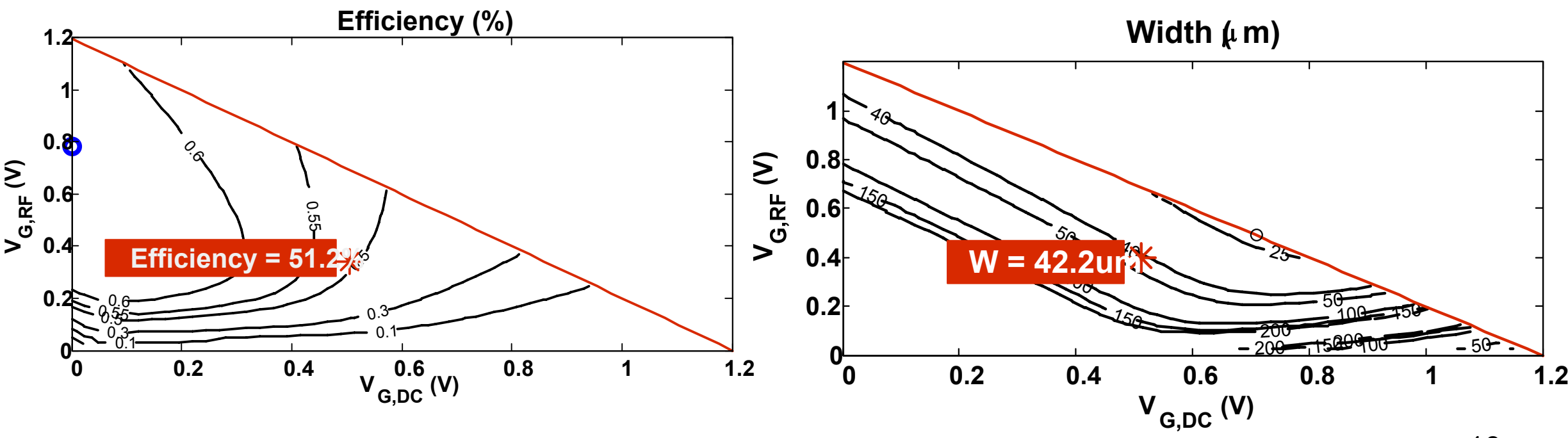


\section{Circuit Implementation (cont'd)}

\begin{tabular}{|l|c|}
\hline Characteristic & $\begin{array}{c}\text { Post-layout } \\
\text { simulations }\end{array}$ \\
\hline $\mathrm{V}_{\mathrm{DD}}(\mathrm{V})$ & 0.65 \\
\hline $\mathrm{P}_{\mathrm{DC}}(\mathrm{mW})$ & 3.16 \\
\hline$\eta(\%)$ & 46.6 \\
\hline$P_{\text {out }}(\mathrm{dBm})$ & 1.9 \\
\hline$G_{\text {pow }}(\mathrm{dB})$ & 26 \\
\hline$\hat{b}_{\mathrm{bC}}(\mathrm{mA})$ & 4.6 \\
\hline$P_{\text {harm }}^{2 \text { nd }}(\mathrm{dBc})$ & -67 \\
\hline$P_{\text {harm }}^{3 \mathrm{rd}}(\mathrm{dBc})$ & -21 \\
\hline
\end{tabular}




\section{Circuit Implementation (cont'd)}

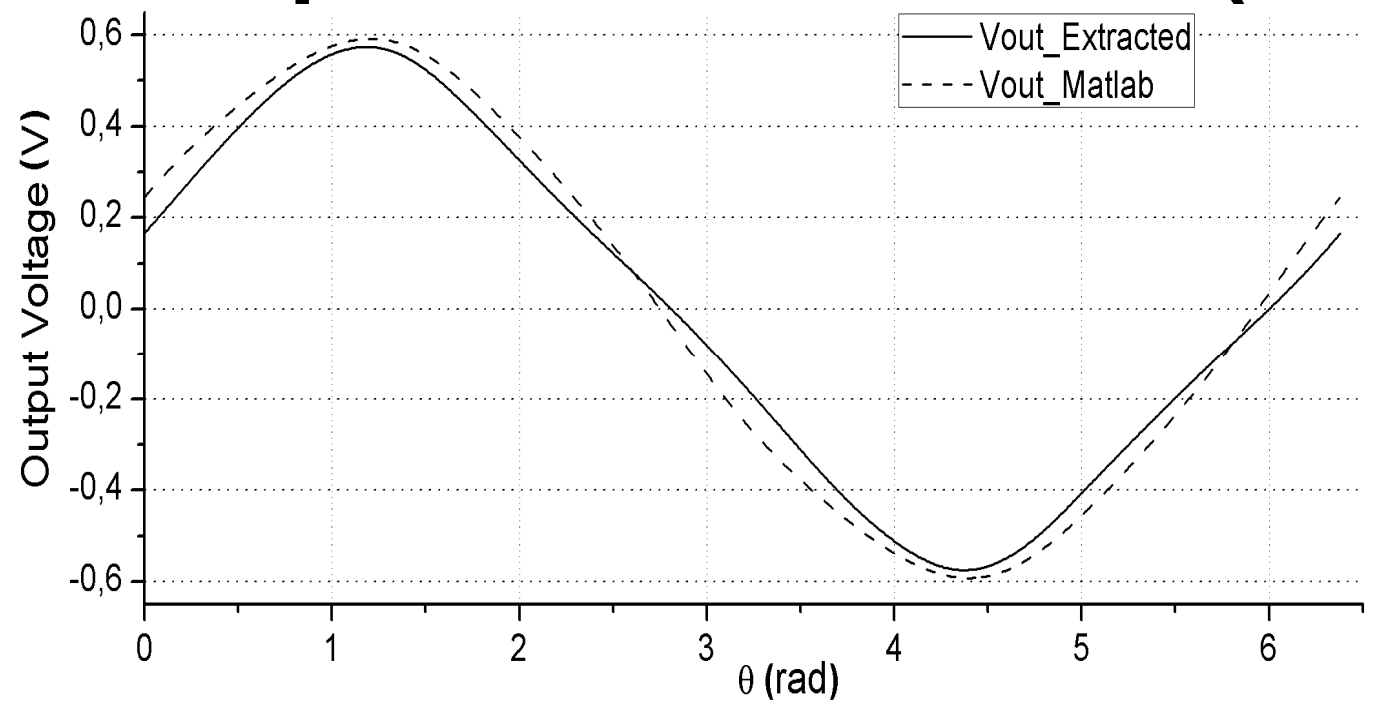

Output voltage

$\overline{\mathrm{P}}_{\text {out }}=1.92 \mathrm{dBm}$
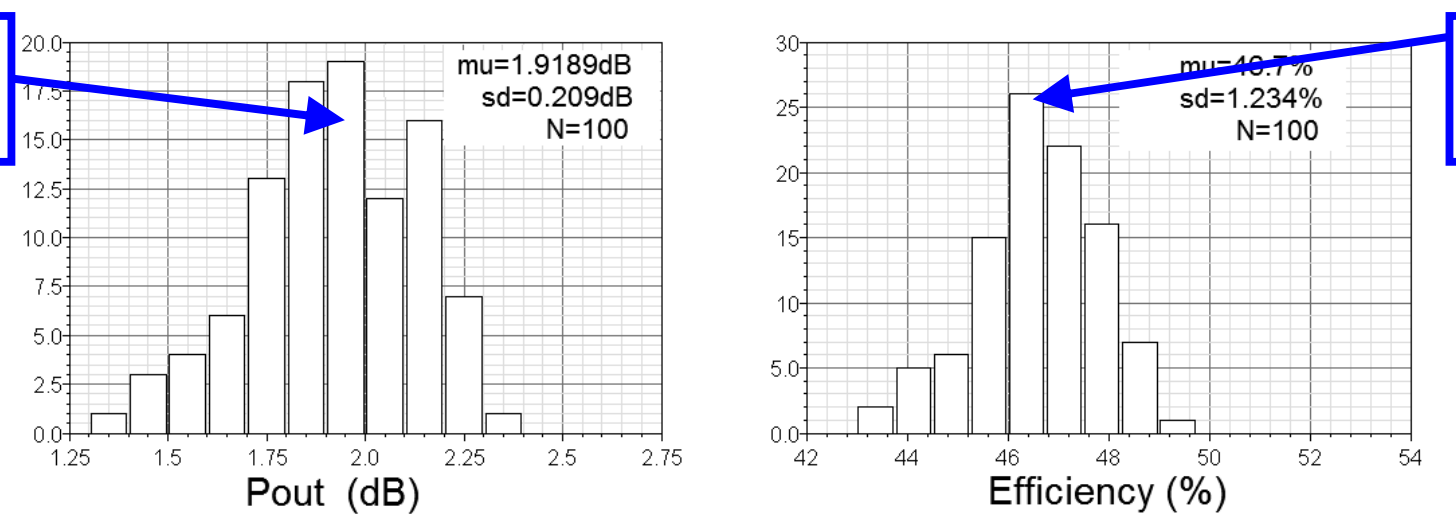

$\bar{\eta}=46.7 \%$

Montecarlo results for $\mathrm{P}_{\text {out }}$ and PA efficiency. 


\section{Comparison with other works}

\begin{tabular}{|l|l|l|l|l|l|l|l|}
\hline Ref & $\begin{array}{l}\text { Techno } \\
(\mathrm{nm})\end{array}$ & PA class & $\begin{array}{l}\mathrm{P}_{\text {out }} \\
(\mathrm{mW})\end{array}$ & $\begin{array}{l}\mathrm{G}_{\text {pow }} \\
(\mathrm{dB})\end{array}$ & $\begin{array}{l}\text { PAE } \\
(\%)\end{array}$ & $\begin{array}{l}\text { freq. } \\
(\mathrm{GHz})\end{array}$ & $\begin{array}{l}\text { FOM } \\
\left(\mathrm{W} \times \mathrm{GHz}^{2}\right)\end{array}$ \\
\hline$[2]$ & 180 & $\mathrm{AB}$ & 2.2 & 8 & 14 & 2.4 & 1.45 \\
\hline$[3]$ & 350 & $\begin{array}{l}\text { Casdode } \\
+ \text { Class C }\end{array}$ & 1 & 15 & 33 & 2.4 & 2.9 \\
\hline$[4]$ & 350 & $\begin{array}{l}\text { Class A + } \\
\text { Class C }\end{array}$ & 3.1 & 19 & 14 & 2.4 & 4.8 \\
\hline$[5]$ & 180 & $\begin{array}{l}\text { w/Folded } \\
\text { Cascode }\end{array}$ & 1 & 12 & 18.5 & 2.4 & 1.3 \\
\hline & 90 & Class C & 1.5 & 25.7 & 46.5 & 2.445 & 10.7 \\
\hline
\end{tabular}

[2] Khannur RFIC 2003

[3] Choi JSSC 2003

[4] Zito ICECS 2006

FoM $=P_{\text {out }} \cdot G_{\text {pow }} \cdot P A E \cdot$ freq $^{2}$

[5] Nguyen TMTT 2006

(ITRS roadmap 2009 system drivers). 


\section{Conclusions}

A compatible IEEE802.15.4 Class C PA design in 90nm CMOS is presented.

It is based on the "Design efficiency approach" presented in [1]

Post layout results and preliminary measurements show very good agreement with Matlab results.

Final design reaches interesting efficiency values and surpass the output power specification of the standard. 


\section{A Fully Differential Monolithic 2.4GHz PA for IEEE 802.15.4 based on Efficiency Design Flow}

Rafaella Fiorelli Eduardo Peralías

IMSE-CNM CSIC-Univ. de Sevilla España
Nicolás Barabino Fernando Silveira
GME-Univ. de la República Uruguay

Contact: fiorelli@imse-cnm.csic.es 